\title{
Stirling Engine and absolute sealing
}

\author{
Thierry Raballand ${ }^{1}$ \\ ${ }^{1} 22$ rue de Ouagadougou, 33300 Bordeaux, France
}

\begin{abstract}
Referring to the sketches, the alternative rotation of shaft (101) allows for absolute sealing between the left side and the right side of seal (1020). A device for total sealing around a shaft in alternating rotation is characterized in that a seal (1020), the main part of the device, called a torsion disc, constitutes the material insulator and is fixed on a shaft (101) by means of two spacers (610) and (620), where the spacer (610) is held in position by the bearing (710) and the spacer (620), which clamps the seal (1020), is held in position by the bearing (720). Along the shaft (101) on both sides of the seal (1020), O-rings (510) and (520) and possibly temperature insulating rings (410) and (420) can be arranged. On both sides of the seal (1020) there are possibly two pressure isolating stops (210) and (220), which slide in rotation on two other stops (110) and (120). The two bearing housings (228) and (8) are positioned relative to each other by means of shims (7) in order to clamp the seal (1020) at the bore and at the shaft. Connecting screws connect the bearing housings (228) and (8) and further connecting screws connect the housing (8) and the wall (22). Absolute sealing of a kinematic Stirling engine is now achievable. A French patent was taken out on August 27th, 2020.
\end{abstract}




\section{PROBLEM}

\subsection{Need for seal}

Absolute sealing is the main problem of Stirling engines. A powerful engine requires high pressure. So a strong seal is needed.

\subsection{Triple seal}

A threefold seal is required:

- to get sealing of the working fluid

- to separate the high pressure space from the low pressure space

- to separate the high temperature space from the low temperature space

\section{SOLUTION}

\section{1 "Twistdisk"}

The state of the art proposes a "twistsock", a sock which twists along itself (Patents [1] \& [2]). Here we propose a "twistdisk", a disk which twists around itself.

\subsection{Design of the seal}

As above-mentioned, a threefold seal is required. Details are available in figures Fig.1 and Fig.2. Here follows the layout of the seal:

- a necessary "twistdisk" (1020) to separate the fluid on the left-hand side from the fluid on the right-hand side

- two possible pressure isolating stops (210) and (220), which slide in rotation on two other stops (110) and (120).

- two possible temperature insulating rings (410) and (420).

\section{References}

1. J. Bras, French patent FR2539811 (1983)

2. T. Raballand, French patent FR2846374 (2002) 


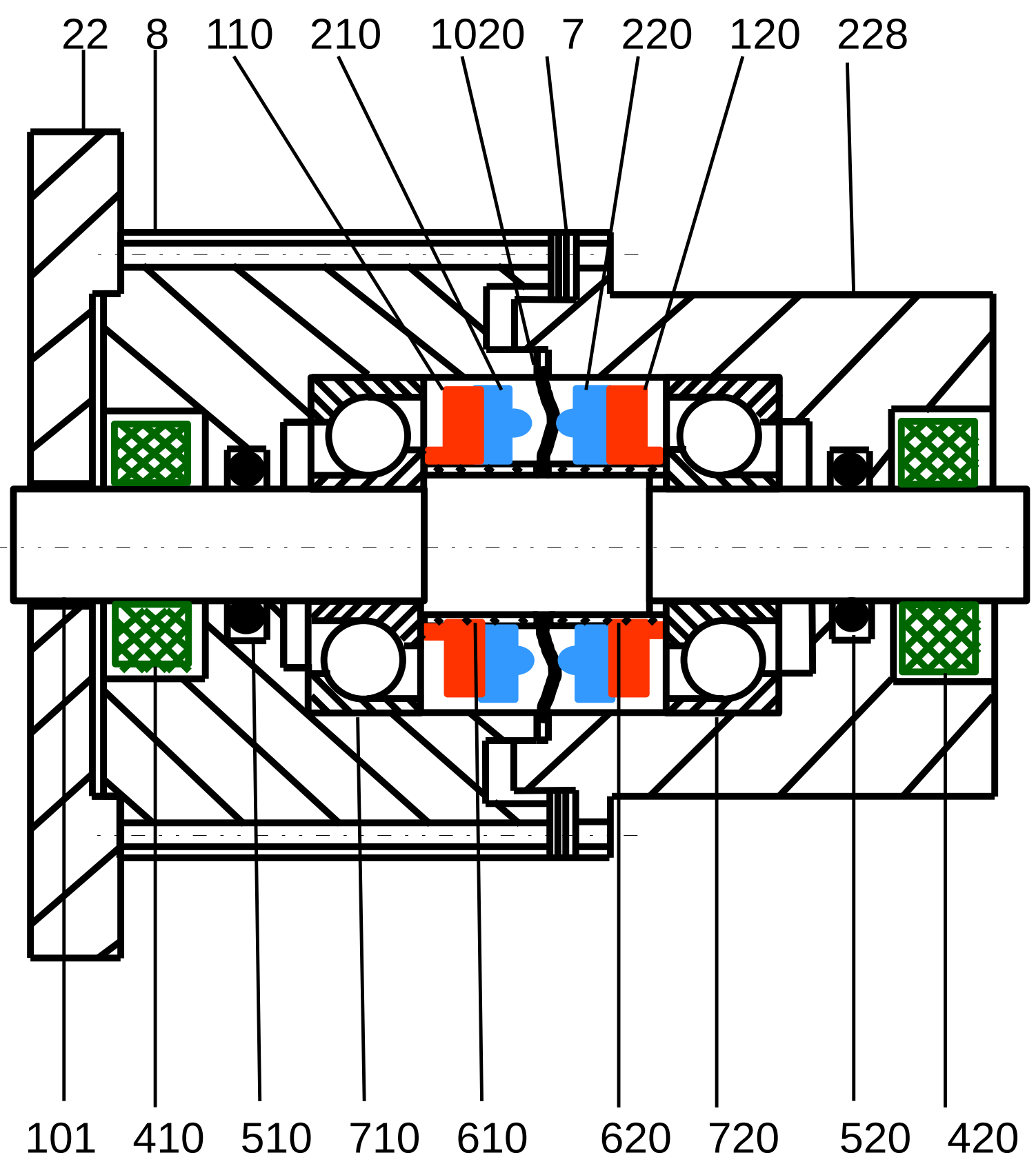

Fig. 1. Twist disk (1020) around alternating shaft (101) enabling absolute sealing 


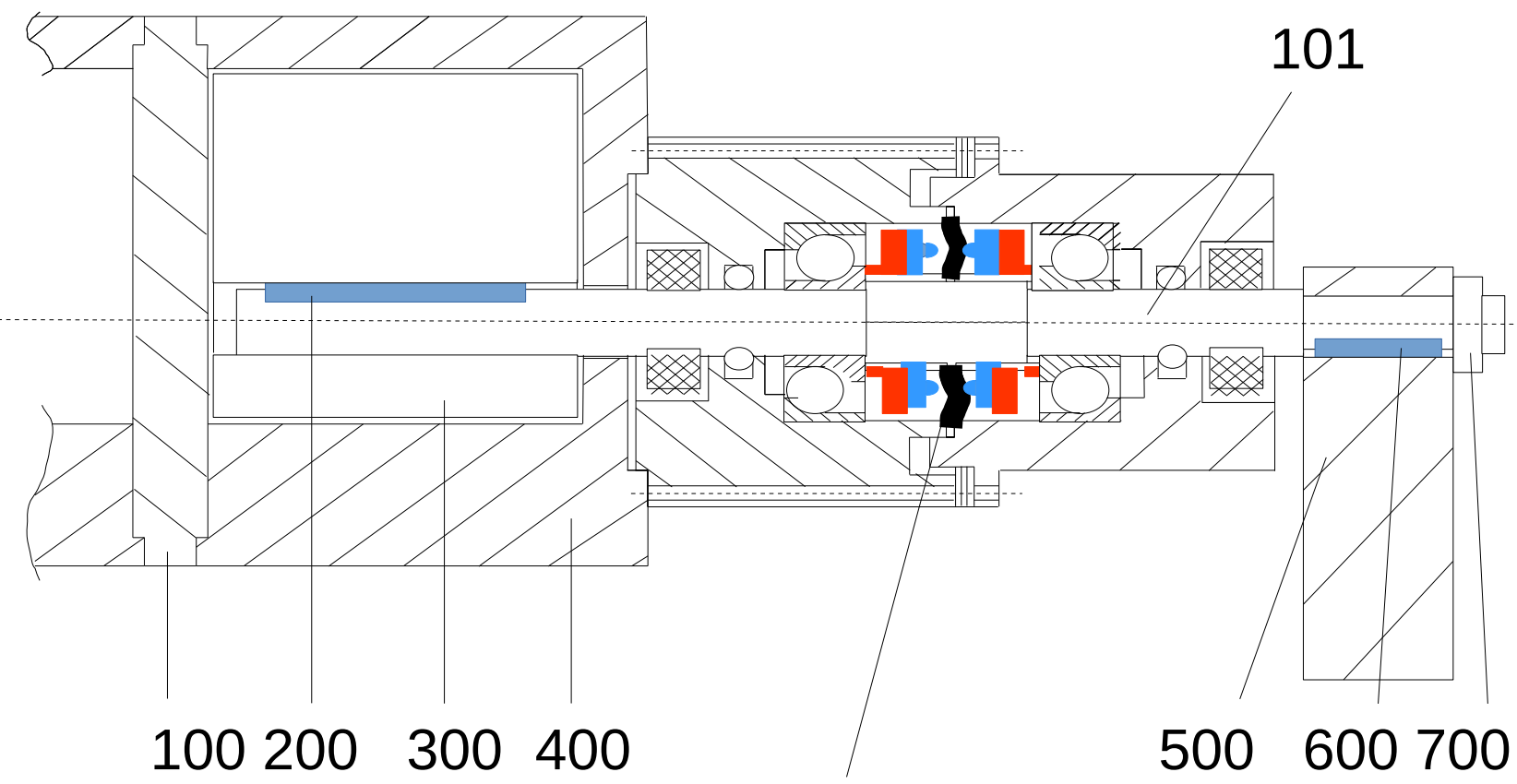

\section{0}

Fig. 2. Twist disk (1020) around alternating shaft (101) enabling absolute sealing for a StirlingFranchot engine with prismatic "flipper" pistons (300) 